\title{
¿Son los criterios de la ASGE suficientes para la estratificación del riesgo de coledocolitiasis?
}

\section{Are the ASGE criteria sufficient to stratify the risk of choledocholithiasis?}

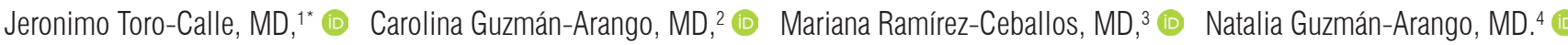

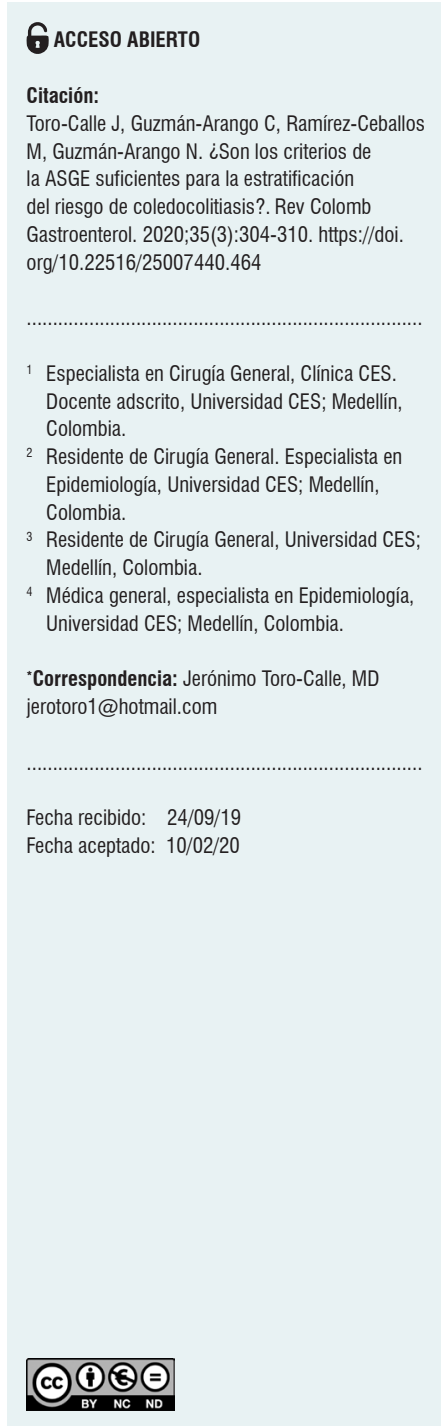

\begin{abstract}
Resumen
Introducción: la patología biliar litiásica es una de las entidades más frecuentes en el área de cirugía general y en gastroenterología. El tratamiento varía según el lugar donde se alojen los cálculos. Para esto, se han definido diversas escalas de estratificación del riesgo de presentar coledocolitiasis, pero son los criterios planteados por la Sociedad Americana de Endoscopia Gastrointestinal (American Society for Gastrointestinal Endoscopy, ASGE) los más usados a nivel mundial, ya que tienen una precisión diagnóstica definida del $70 \%$. Los procedimientos o ayudas diagnósticas establecidas por estos criterios, en ocasiones, prolongan el tiempo de hospitalización, aumentan los costos y pueden tener complicaciones. Metodología: se realizó un estudio observacional analítico, de tipo transversal retrospectivo, con datos obtenidos a partir de las historias clínicas de pacientes sometidos a colecistectomía laparoscópica, en la Clínica CES de Medellín, entre julio y diciembre de 2017. Resultados y conclusiones: se analizaron 424 historias clínicas de pacientes sometidos a colecistectomia laparoscópica. De ellos, $254(56,76 \%)$ se categorizaron como de riesgo bajo, mientras que $94(22,11 \%)$ fueron de riesgo intermedio y $76(17,88 \%)$ de riesgo alto. Se encontró una frecuencia de coledocolitiasis del $90,8 \%$ en aquellos categorizados como de riesgo alto y del $26,6 \%$ en los pacientes de riesgo intermedio. En la categoría de riesgo intermedio se hallaron diferencias estadísticamente significativas entre ambos grupos para los valores de bilirrubina total, bilirrubina directa y aspartato aminotransferasa (AST) $(p=0,001 ; p=0,014 ; p=0,007$, respectivamente). La baja frecuencia de coledocolitiasis en la categoría de riesgo intermedio puede ser explicada por cálculos menores a $5 \mathrm{~mm}$ no visibles en la colangiorresonancia. $A$ partir de este estudio, se propone ajustar los rangos de valores de los criterios de la ASGE para la categoría de riesgo intermedio, permitiendo tener una mayor precisión a la hora de clasificar los pacientes con patología litiásica y disminuir costos y estancia hospitalaria.
\end{abstract}

\section{Palabras clave}

Coledocolitiasis, riesgo de coledocolitiasis, criterios ASGE, colecistitis.

\begin{abstract}
Introduction: Biliary lithiasis is one of the most frequent diseases in the area of general surgery and gastroenterology. Treatment varies depending on the location of the gallstones. Several stratification scales of the risk of choledocholithiasis have been defined, being the criteria proposed by the American Society of Gastrointestinal Endoscopy (ASGE) the most used worldwide, with a diagnostic accuracy of $70 \%$. However, the procedures or diagnostic aids defined by these criteria, sometimes, increase hospital stay, costs, and may lead to the development of complications. Methodology: An observational, analytical, retrospective, crosssectional study was conducted with data obtained from the clinical records of patients undergoing laparoscopic cholecystectomy at the CES Clinic in Medellín, Colombia, between July and December of 2017. Results and conclusions: 424 medical records were analyzed, of which 254 (56.76\%) were classified as low-risk, 94 $(22.11 \%)$ as intermediate-risk and $76(17.88 \%)$ as high-risk. The frequency of choledocholithiasis was $90.8 \%$ in high-risk patients and $26.6 \%$ in intermediate-risk patients. For the intermediate-risk category, statistically
\end{abstract}


significant differences were found between the two groups for the total bilirubin, direct bilirubin, and AST values (p: $0.001, p: 0.014, p: 0.007$, respectively). The low frequency of choledocholithiasis in the intermediaterisk category can be explained by less than $5 \mathrm{~mm}$ gallstones not identified by the cholangioresonance. Based on this study, we propose to adjust the ranges of the ASGE criteria variables for the intermediate-risk category for better accuracy when classifying patients with biliary lithiasis and, thus, reduce costs and hospital stay.

\section{Keywords}

Choledocholithiasis; Choledocholithiasis risk; ASGE criteria; Cholecystitis.

\section{INTRODUCCIÓN}

La patología biliar litiásica es una de las principales causas de consulta en el servicio de cirugía general y en gastroenterología. Esta condición corresponde a un conjunto de enfermedades que afectan la vesícula y las vías biliares intra y extrahepáticas, secundarias a la presencia patológica de cálculos. Dichos cálculos pueden generar impactos en la morbimortalidad del paciente, que son secundarios a la obstrucción de los diferentes conductos, y cuyos síntomas varían desde episodios de dolor abdominal hasta sepsis o muerte $(1,2)$.

Esta patología es una de las entidades más frecuentes y afecta alrededor de 20 millones de personas en el mundo, con prevalencias hasta del $20 \%(1,2)$. De estos pacientes, cerca del $10 \%$ requieren de colecistectomías por causa de sintomatología recurrente, y aproximadamente el $20 \%$ pueden presentar coledocolitiasis en algún momento de la enfermedad.

En Colombia, se realizan aproximadamente 60000 colelitiasis sintomática. Sin embargo, la incidencia de la enfermedad litiásica en el país no está claramente definida, ya que alcanza valores que van desde el $0,3 \%$ hasta el $60 \%$ $(1,3)$.

$\mathrm{Al}$ enfrentar el escenario de un paciente con colelitiasis sintomática o colecistitis es importante clasificar el riesgo de coledocolitiasis, y así poder definir la necesidad de procedimientos o ayudas diagnósticas adicionales (2), como la colangiorrensonancia, ecografía endoscópica o la colangiopancreatografía retrógrada endoscópica (CPRE), que no están exentos de complicaciones $(1,4,5)$.

Por lo anterior, se han creado escalas predictivas, y una de las mundialmente más aceptadas es la que plantea la Sociedad Americana de Endoscopia Gastrointestinal (American Society for Gastrointestinal Endoscopy, ASGE), elaborada en 2010. En ella se propone estratificar a los pacientes según el riesgo (bajo, intermedio o alto) de presentar coledocolitiasis. Este riesgo se calcula a partir de imágenes, pruebas de función hepática y factores asociados (Tabla 1).

Tabla 1. Predictores de coledocolitiasis ASGE, 2010

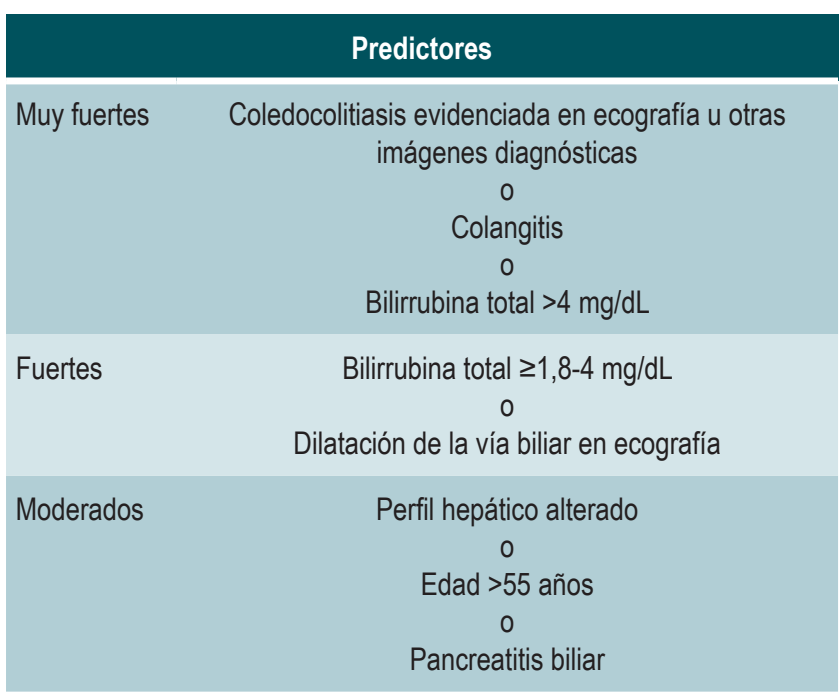

ASGE: Sociedad Americana de Endoscopia Gastrointestinal (American Society for Gastrointestinal Endoscopy).

Una vez se tiene esta categorización, se realiza una de las siguientes intervenciones: colecistectomía laparoscópica, colangiografía por resonancia magnética, ecografía endoscópica o CPRE $(6,7)$. En la actualidad, estos criterios tienen una precisión diagnóstica cercana al $70 \%(8)$.

Sin embargo, aun aplicando estos criterios, los procedimientos o ayudas diagnósticas definidos tienen complicaciones y altos costos. Por lo tanto, es importante establecer con mayor precisión el riesgo de cada paciente de coledocolitiasis y posteriomente la realización de la ayuda diagnóstica o del procedimiento indicado, e impactar así en la disminución de la morbimortalidad y en los costos del sistema de salud. 
El objetivo de este estudio es describir las características demográficas, clínicas, de ayudas diagnósticas y los hallazgos quirúrgicos en los pacientes con patología litiásica biliar, en una institución de tercer nivel. Para ello se determinó la frecuencia de coledocolitiasis en cada una de las categorías de riesgo, según los criterios de la ASGE, y se identificaron los factores asociados a la coledocolitiasis en los pacientes con riesgo intermedio.

\section{MATERIALES Y MÉTODOS}

Este es un estudio observacional, tipo transversal, retrospectivo, con interés analítico. Para su desarrollo, se revisaron las historias clínicas de los pacientes a quienes se les realizó una colecistectomía laparoscópica por patología litiásica biliar, entre julio y diciembre de 2017, en una clínica privada de tercer nivel. Esta clínica cuenta con el servicio de cirugía general y en ella se realizan mensualmente, en promedio, 130 colecistectomías por vía laparoscópica.

Luego de realizarse la colecistectomía laparoscópica, se recolectó información sobre la estratificación del riesgo de coledocolitiasis, según los criterios de la ASGE de 2010 (Tabla 1). De acuerdo con estos parámetros, los pacientes categorizados en riesgo bajo fueron llevados directamente a colecistectomía por laparoscopia, mientras que a aquellos de riesgo intermedio se les realizó una colangiorresonancia. Según el resultado de esta última, fuera negativa o positiva para coledocolitiasis, los pacientes fueron conducidos a una colecistectomía laparoscópica o a una CPRE mas colecistectomía laparoscópica en el mismo acto quirúrgico, respectivamente.

Entre tanto, a los pacientes de alto riesgo se les realizó una CPRE.

Se excluyeron los pacientes con patología biliar maligna, diagnóstico de coledocolitiasis recurrente, antecedente de intervenciones de la vía biliar o derivaciones biliares, así como con un diagnóstico de disquinesia biliar o enfermedad hepática previa. Además, se excluyeron las historias clínicas que no contaran con la totalidad de las variables para calcular el riesgo de coledocolitiasis, según los criterios de la ASGE.

Dentro de ese contexto, se realizó el análisis univariado, calculando las frecuencias absolutas y relativas de las variables cualitativas y las medianas con rango intercuartílico de las variables cuantitativas. Para comparar los grupos (coledocolitiasis frente a no coledocolitiasis) en los pacientes de riesgo intermedio, se utilizó una prueba de chi-cuadrado $\left(\chi^{2}\right)$ o la prueba de $U$ de Mann Whitney. Asimismo, se consideró significativo un valor de $p \leq 0,05$. Los datos se analizaron en el programa estadístico SPSS $22^{\circledR}$.

\section{RESULTADOS}

Entre julio y diciembre de 2017, 467 pacientes con patología litiásica de la vesícula biliar fueron sometidos a una colecistectomía laparoscópica en la Clínica CES de Medellín. Después de aplicar criterios de exclusión, se analizaron 424 personas con esta enfermedad (Figura 1). Así, $76(17,8 \%)$ de los pacientes fueron clasificados en riesgo alto, $94(22,1 \%)$ en riesgo intermedio y $254(59,7 \%)$ pacientes en riesgo bajo. Estos enfermos fueron manejados con CPRE, colangiorresonancia, y colecistectomía por laparoscopia, respectivamente.

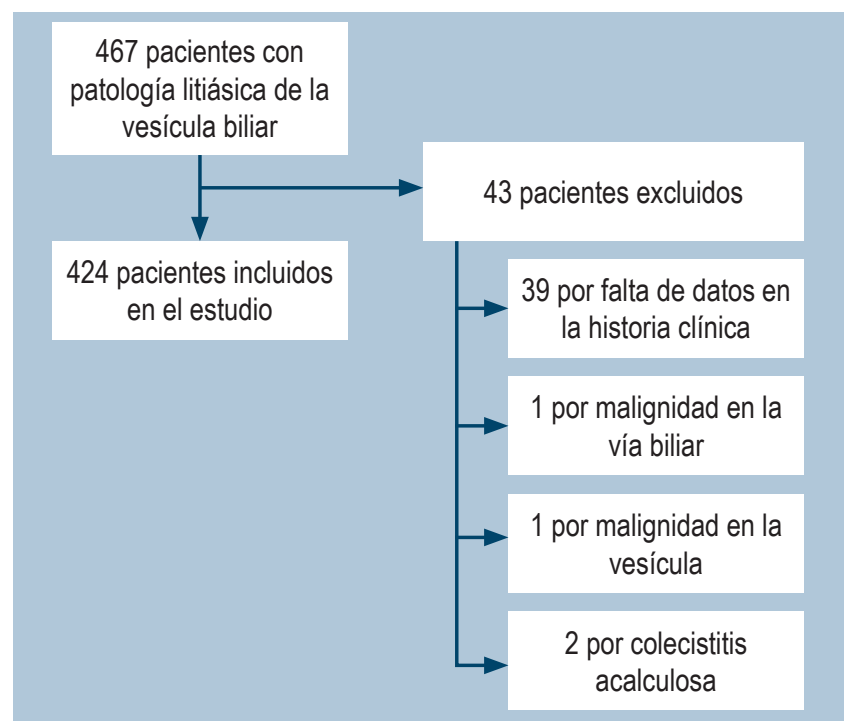

Figura 1. Flujograma de selección de pacientes en el estudio.

Se identificó una mediana de edad de los pacientes con coledocolitiasis similar a la de aquellas personas sin dicho diagnóstico (45 frente a 41 años $p=0,114$ ); en ambos grupos predominó el sexo femenino ( 77 y $69 \%$, respectivamente; $p=0,118$ ). El desarrollo de pancreatitis aguda y colangitis fue mayor en los pacientes con coledocolitiasis, en comparación con aquellos sin coledocolitiasis (17\% frente al 7,3\%; $p=0,004 ; 13,8 \%$ frente al $0,6 \% ; p<0,0001$ ). La presencia de colecistitis aguda fue mayor en el grupo sin coledocolitiasis ( $77 \%$ frente al 59,9\%; $p=0,001$ ). Todos los pacientes con síndrome de Mirizzi pertenecieron al grupo sin coledocolitiasis (Tabla 2).

Al comparar los paraclínicos de ambos grupos de pacientes, no se encontraron diferencias en los valores para el hemograma y los reactantes de fase aguda. Sin embargo, se observa una mayor frecuencia del patrón colestásico en los pacientes con coledocolitiasis. Además, se evidencia un aumento en los valores de la bilirrubina total, la bilirrubina 
Tabla 2. Características de la población con patología biliar litiásica

\begin{tabular}{|c|c|c|c|}
\hline & $\begin{array}{l}\text { Coledocolitiasis } \\
\qquad n=94\end{array}$ & $\begin{array}{l}\text { No coledocolitiasis } \\
\qquad n=330\end{array}$ & p \\
\hline \multicolumn{4}{|l|}{ Características demográficas } \\
\hline Edad (Me-RIQ) & $45(32-61)$ & $41(30-53)$ & 0,114 \\
\hline Sexo femenino (n [\%]) & $73(77)$ & $229(69)$ & 0,118 \\
\hline Pancreatitis aguda (n [\%]) & $16(17)$ & $24(7,3)$ & 0,004 \\
\hline Historia de pancreatitis (n [\%]) & 0 & $3(0,9)$ & 1 \\
\hline Colangitis (n [\%]) & $13(13,8)$ & $2(0,6)$ & $<0,0001$ \\
\hline Colecistitis [n (\%)] & $56(59,6)$ & $254(77)$ & 0,001 \\
\hline Síndrome de Mirizzi (n [\%]) & 0 & $5(1,5)$ & 0,591 \\
\hline \multicolumn{4}{|l|}{ Laboratorios } \\
\hline Hemoglobina (Me-RIQ) & $13,6(12,3-14,6)$ & $13,5(12,7-14,6)$ & 0,473 \\
\hline Leucocitos (Me-RIQ) & $10150(7753-13888)$ & $10070(7270-14$ 135) & 0,398 \\
\hline PCR (Me-RIQ) & $5,15(0,96-21)$ & $3,17(0,5-13,3)$ & 0,028 \\
\hline Bilirrubina total (Me-RIQ) & $3,9(1,6-5,1)$ & $0,64(0,42-1,1)$ & $<0,0001$ \\
\hline Bilirrubina directa (Me-RIQ) & $2,5(1-4,2)$ & $0,32(0,2-0,56)$ & $<0,0001$ \\
\hline AST (Me-RIQ) & $226(131,5-355,1)$ & $26(18,7-47,5)$ & $<0,0001$ \\
\hline ALT (Me-RIQ) & $261(151,8-410)$ & $30,6(18,4-67)$ & $<0,0001$ \\
\hline Fosfatasa alcalina (Me-RIQ) & $223(155,8-364,3)$ & $97(72-162)$ & $<0,0001$ \\
\hline GGT (Me-RIQ) & $570,5(269,3-770)$ & $80,5(46,3-258)$ & $<0,0001$ \\
\hline \multicolumn{4}{|l|}{ Imágenes } \\
\hline \multicolumn{4}{|l|}{ Ecografía } \\
\hline Dilatación del colédoco (n [\%]) & $58(61,7)$ & $13(3,9)$ & $<0,0001$ \\
\hline Cálculo en colédoco (n [\%]) & $35(37,2)$ & $1(0,3)$ & $<0,0001$ \\
\hline Colangiorresonancia & & & $<0,0001$ \\
\hline Colecistocoledocolitiasis (n [\%]) & $29(100)$ & 0 & \\
\hline Sin cálculos en la vía biliar (n [\%]) & 0 & $50(100)$ & \\
\hline \multicolumn{4}{|l|}{ CPRE } \\
\hline Coledocolitiasis (n [\%]) & $94(100)$ & 0 & $<0,001$ \\
\hline Sin cálculos en la vía biliar (n [\%]) & 0 & $6(66,6)$ & \\
\hline Estenosis de la vía biliar (n [\%]) & 0 & $1(11,1)$ & \\
\hline Hepatolitiasis (n [\%]) & 0 & $2(22,2)$ & \\
\hline \multicolumn{4}{|l|}{ Colecistectomía por laparoscopia } \\
\hline Tiempo de cirugía (Me-RIQ) & $60(40-120)$ & $60(47,5-75,5)$ & 0,128 \\
\hline Conversión a abierta (n [\%]) & $2(2,1)$ & $2(0,6)$ & 0,214 \\
\hline Lesión de vía biliar (n [\%]) & $1(1,1)$ & $2(0,6)$ & 0,529 \\
\hline Total (n [\%]) & $94(22)$ & $330(78)$ & \\
\hline
\end{tabular}

ASGE: Sociedad Americana de Endoscopia Gastrointestinal (American Society for Gastrointestinal Endoscopy); RIQ: rango intercuartílico; ME: mediana; n: número; PCR: proteína C-reactiva; AST: aspartato aminotransferasa; ALT: alanina aminotransferasa; GGT: $\gamma$-glutamil transpeptidasa; CPRE: colangiopancreatografía retrógrada endoscópica 
directa, $\gamma$-glutamil transpeptidasa (GGT), la fosfatasa alcalina y las transaminasas en este grupo (Tabla 2).

Asimismo, la dilatación de la vía biliar $(>6 \mathrm{~mm})$ y la presencia de imágenes hipoecoicas compatibles con cálculos en la vía biliar, evidenciados en la ecografía, fueron más frecuentes en los pacientes con coledocolitiasis $(61,7 \%$ frente al 3,9 \% y $37,2 \%$ frente al 0,3 \%, respectivamente; todos con una $p<0,0001)$. En adición, 103 pacientes fueron conducidos a una CPRE. De ellos, 94 (91,3\%) tuvieron el diagnóstico confirmado de coledocolitiasis, mientras que $9(8,7 \%)$ presentaron diagnósticos diferentes: 6 sin evidencia de cálculos, 1 de estenosis de la vía biliar y 2 de hepatolitiasis. Los tiempos del procedimiento quirúrgico, el riesgo de conversión y la lesión de la vía biliar no difirieron en ambos grupos (Tabla 2).

$\mathrm{Al}$ realizar la categorización del riesgo de coledocolitiasis se evidenció, a través de una CPRE, que los pacientes que se clasificaron como alto riesgo tuvieron una frecuencia del 90,8 \% de presentación de la patología. Por otro lado, en los pacientes identificados como riesgo intermedio, se encontró, mediante la colangiorresonancia, que el 26,6\% de ellos presentaban una coledocolitiasis, y hasta el 73,4 \% solo tenían colelitiasis.

La totalidad de los pacientes cuya colangiorresonancia reportó una coledocolitiasis fueron conducidos a una CPRE terapéutica, en la cual se confirmó el diagnóstico en el $100 \%$ de los casos. Para este grupo (riesgo intermedio, según la ASGE), los valores de bilirrubina total, bilirrubina directa y AST diferían entre quienes tenían o no coledocolitiasis ( $p=0,001 ; p=0,014 ; p=0,007$, respectivamente) (Tabla 3$)$.

\section{DISCUSIÓN}

En la actualidad, la enfermedad litiásica biliar es una condición claramente identificada, que obliga al médico general, al gastroenterólogo y al cirujano general, a tener un enfoque diagnóstico adecuado. Esta patología contempla un espectro de manifestaciones clínicas y paraclínicas según la localización de la litiasis, y hasta el día de hoy no existe un enfoque óptimo para los pacientes con riesgo de coledocolitiasis.

Dentro de la literatura, se encuentra una variedad de modelos predictivos que pretenden enfocar el manejo óptimo de estos pacientes. La escala de la ASGE estratifica a dichos pacientes según criterios clínicos, imagenológicos por ecografía y laboratorios, y los categoriza en tres subgrupos diferentes, con el fin de realizar un manejo basado en el riesgo de coledocolitiasis.

Desde la publicación de la guía de la ASGE de 2010, se han realizado varios estudios que valoran el rendimiento de los criterios en diferentes poblaciones. En este sentido, se han encontrado tasas de rendimiento de las categorías de alto riesgo del 75,59 \% y un riesgo intermedio del 49,35\% (9). Asimismo, existen otros estudios retrospectivos, basados en la CPRE como prueba de referencia, que demuestran valores predictivos para la categoría de alto riesgo,

Tabla 3. Criterios ASGE en pacientes con riesgo intermedio según la presentación de coledocolitiasis

\begin{tabular}{lccc} 
& Coledocolitiasis & No coledocolitiasis & $p$ \\
\hline Edad (Me-RIQ) & $41(30,5-59,5)$ & $42(32,5-53,5)$ & 0,64 \\
\hline Pancreatitis aguda $(\mathrm{n}[\%])$ & $4(16)$ & $17(24,6)$ & 0,37 \\
Historia de pancreatitis $(\mathrm{n}[\%])$ & $0(0)$ & $1(1,4)$ & 0,73 \\
\hline Colecistitis $(\mathrm{n}[\%])$ & $15(60)$ & $47(68,1)$ & 0,46 \\
\hline Bilirrubina total (Me-RIQ) & $1,94(1,55-3,97)$ & $1,27(0,62-2,16)$ & 0,001 \\
Bilirrubina directa (Me-RIQ) & $1,76(0,91-2,69)$ & $0,74(0,27-1,75)$ & 0,014 \\
AST (Me-RIQ) & $266(162,5-500)$ & $112(35-380)$ & 0,007 \\
ALT (Me-RIQ) & $297(147,5-386,5)$ & $129(60-450)$ & 0,223 \\
Fosfatasa alcalina (Me-RIQ) & $157(131-267,5)$ & $185(135-242)$ & 0,76 \\
GGT (Me-RIQ) & $537(272-576)$ & $368(208-603)$ & 0,286 \\
Dilatación del colédoco (>6 mm) (n [\%]) & $3(12)$ & $9(13)$ & 0,6
\end{tabular}

RIQ: rango intercuartílico; ME: mediana; n: número; AST: aspartato aminotransferasa; ALT: alanina aminotransferasa; GGT: $\gamma$-glutamil transpeptidasa 
entre el 70 y el $90 \%$, y para la categoría de riesgo intermedio, entre el 34 y el $50 \%(10,11)$.

$\mathrm{Al}$ analizar los datos obtenidos, se evidencia que la frecuencia de coledocolitiasis en las categorías de alto y bajo riesgo es muy similar a la encontrada en la literatura internacional, con resultados que generan confiabilidad en el enfoque. En contraste con la literatura, los pacientes categorizados en riesgo intermedio alcanzan una frecuencia del $25 \%$ para padecer coledocolitiasis.

Estos criterios ayudan al clínico a descartar la enfermedad, pero no tiene exactitud a la hora de confirmarla, lo que genera un sobreuso de la colangiorresonancia, cuyos resultados, en su mayoría, serán negativos. Dicho escenario aumentará los costos y el tiempo de hospitalización en este tipo de pacientes. Además, hay que tener en cuenta que esta ayuda diagnóstica no tiene disponibilidad de rutina en todos los centros de atención.

Es por tal razón que se analizó específicamente cuáles variables del riesgo intermedio podían asociarse a la presencia de coledocolitiasis, y se halló que los valores superiores de bilirrubina total de $1,94 \mathrm{mg} / \mathrm{dL}$, bilirrubina directa de $1,76 \mathrm{mg} / \mathrm{dL}$ y AST de $266 \mathrm{mg} / \mathrm{dL}$ tienen una asociación estadísticamente significativa.

En la literatura, varios artículos describen la utilidad del uso del valor de la bilirrubina total y de la fosfatasa alcalina como predictores de la coledocolitiasis $(11,12)$.

En los pacientes de riesgo intermedio, los hallazgos ecográficos no mostraron una asociación significativa con la presencia de coledocolitiasis. Sin embargo, la medida de la dilatación del colédoco debe interpretarse según las características de cada paciente, como la edad y el índice de masa corporal (13).

En agosto de 2019, se realizó una actualización de los criterios de la ASGE (14). Entre las modificaciones principales, se retira la historia o la presencia de pancreatitis aguda y se agregan como señal de alto riesgo la dilatación de la vía biliar $>6 \mathrm{~mm}$, conjuntamente con la bilirrubina total $>4 \mathrm{mg} / \mathrm{dL}$. Además, se deja la bilirrubina $>4 \mathrm{mg} / \mathrm{dL}$ de manera aislada como un predictor de riesgo intermedio.

Como limitación de este estudio, es necesario anotar que no se pudo contar con un método de referencia (gold standard) único para definir la presencia o ausencia de coledocolitiasis. La literatura reporta diferentes ayudas diagnósticas para el riesgo intermedio (colangiorresonancia, ultrasonido endoscópico o colangiografía intraoperatoria). No obstante, las últimas dos mencionadas no están disponibles rutinariamente en la institución. Esto impide darle a este estudio un alcance de pruebas diagnósticas.

Es importante señalar que no todas las pruebas diagnósticas sugeridas por las guías de la ASGE tienen una adecuada disponibilidad en nuestro medio. El ultrasonido endoscó- pico es una de las pruebas más escasas y solo se cuenta con acceso en hospitales de cuarto nivel en nuestra ciudad. Por otro lado, aunque podría decirse que la colangiorresonancia es el método de mayor disponibilidad, aún se mantienen grandes barreras sociales y económicas que nos impiden su libre realización cuando es pertinente.

En este estudio, los pacientes de riesgo alto y bajo para coledocolitiasis alcanzan una precisión diagnóstica acorde con lo propuesto por la ASGE, lo que implica la realización de CPRE y colecistectomía por laparoscopia, sin necesidad de estudios adicionales. Se considera que, aunque la aplicación de la guía de la ASGE contribuye a elaborar un enfoque de los pacientes con un modelo de riesgo, es necesaria la búsqueda de alternativas que permitan identificar mejor a quienes requieren verdaderamente el uso de pruebas diagnósticas costosas y de difícil acceso como la colangiorresonancia.

Se propone entonces, según los resultados de este estudio, realizar investigaciones adicionales que permitan evaluar el comportamiento y la utilidad de las pruebas de perfil hepático para identificar la presencia de coledocolitiasis. Entre estas pruebas se hallan los marcadores independientes, principalmente de la bilirrubina total, la bilirrubina directa y AST como variables para los pacientes de riesgo intermedio. Asimismo, se plantea buscar puntos de corte específicos para cada uno de ellos. Dichos estudios deberán desarrollarse basados en los nuevos criterios de la ASGE de 2019, dado que estos cambiarán el número de falsos positivos detectados.

\section{CONCLUSIÓN}

Este estudio confirma que los pacientes con coledocolitiasis demuestran diferencias en sus pruebas de función hepática, reactantes de fase aguda e imagenológicas, al compararlos con las personas que no presentan esta entidad. De igual modo, se observa que los predictores de coledocolitiasis y las categorías de riesgo propuestos por la ASGE en 2010 permiten predecir de manera adecuada la presencia de coledocolitiasis en las clasificaciones de alto y bajo riesgo.

Sin embargo, según nuestros hallazgos, los pacientes categorizados en riesgo intermedio alcanzan una frecuencia del $25 \%$ de coledocolitiasis, lo que constituye una gran ayuda para el personal médico a la hora de descartar la enfermedad, pero no para confirmarla. La baja frecuencia de coledocolitiasis en la categoría de riesgo intermedio puede ser explicada, mediante colangiorresonancia, por la existencia de cálculos $<5 \mathrm{~mm}$, para lo cual podría ser de gran utilidad el uso de la ultrasonografía endoscópica. Por lo anterior, es necesario tomar esta investigación como un trabajo inicial a fin de buscar estrategias más precisas para las predicciones de coledocolitiasis. 


\section{Agradecimientos}

A la Clínica CES y a la Universidad CES por su apoyo en la elaboración de este estudio; también al Dr. José Hugo Arias
Botero por la revisión y el asesoramiento metodológico de este trabajo.

\section{REFERENCIAS}

1. Quintanilla C, Flisfisch H. Coledocolitiasis. Rev Medicina y Humanidades. 2009;1(3):160-168.

2. Oblitas NM. Pruebas de laboratorio como predictores de coledocolitiasis en pacientes sometidos a CPRE en un hospital nacional de referencia. Lima: Facultad de Medicina, Universidad Nacional Mayor de San Marcos; 2015.

3. Almora CL, Arteaga Y, Plaza T, Prieto Y, Hernández Z. Diagnóstico clínico y epidemiológico de la litiasis vesicular. Revisión bibliográfica. Rev Ciencias Médicas. 2012;16(1):200-214.

4. Wilkins T, Agabin E, Varghese J, Talukder A. Gallbladder Dysfunction: Cholecystitis, Choledocholithiasis, Cholangitis, and Biliary Dyskinesia. Prim Care. 2017;44(4):575-597. http://doi.org/10.1016/j.pop.2017.07.002

5. Gómez M, Pion J, Otero W. Predictores de coledocolitiasis en pacientes sometidos a colangiografía retrógrada endoscópica en el Hospital El Tunal de Bogotá. Rev Col Gastroenterol. 2011;26(4):243-252.

6. ASGE Standards of Practice Committee, Maple JT, BenMenachem T, Anderson MA, Appalaneni V, Banerjee S, Cash BD, Fisher L, Harrison ME, Fanelli RD, Fukami N, Ikenberry SO, Jain R, Khan K, Krinsky ML, Strohmeyer $\mathrm{L}$, Dominitz JA.. The role of endoscopy in the evaluation of suspected choledocholithiasis. Gastrointest Endosc. 2010;71(1):1-9.

http://doi.org/10.1016/j.gie.2009.09.041

7. Abuhadba NE. Asociación entre predictores de coledocolitiasis diagnosticada por colangiografía intraoperatoria en el Hospital Nacional Arzobispo Loayza. Lima: Universidad de San Martín de Porres; 2016.

8. Sethi S, Wang F, Korson AS, Krishnan S, Berzin TM, Chuttani R, Pleskow DK, Sawhney MS. Prospective assessment of consensus criteria for evaluation of patients with suspected choledocholithiasis. Dig Endosc. 2016;28(1):75-82. http://doi.org/10.1111/den.12506
9. Sethi S, Krishnan S, Korson AS, Chuttani R, Pleskow DK, Berzin TM, Cury M, Wang F, Sawhney M, Whadhwa V. Prospective validation of ASGE criteria for the evaluation of suspected choledocholithiasis [abstract]. J Gastroenterol Hepatol. 2013;28 Suppl 3:18.

10. Rubin MI, Thosani NC, Tanikella R, Wolf DS, Fallon MB, Lukens FJ. Endoscopic retrograde cholangiopancreatography for suspected choledocholithiasis: testing the current guidelines. Dig Liver Dis. 2013;45(9):744-749. http://doi.org/10.1016/j.dld.2013.02.005

11. Magalhães J, Rosa B, Cotter J. Endoscopic retrograde cholangiopancreatography for suspected choledocholithiasis: From guidelines to clinical practice. World J Gastrointest Endosc. 2015;7(2):128-134. http://doi.org/10.4253/wjge.v7.i2.128

12. Al-Jiffry BO, Elfateh A, Chundrigar T, Othman B, Almalki O, Rayza F, Niyaz H, Elmakhzangy H, Hatem M. Noninvasive assessment of choledocholithiasis in patients with gallstones and abnormal liver function. World J Gastroenterol. 2013;19(35):5877-82. http://doi.org/10.3748/wjg.v19.i35.5877

13. Caddy GR, Tham TC. Gallstone disease: Symptoms, diagnosis and endoscopic management of common bile duct stones. Best Pract Res Clin Gastroenterol. 2006;20(6):1085-1101. http://doi.org/10.1016/j.bpg.2006.03.002

14. ASGE Standards of Practice Committee, Buxbaum JL, Abbas Fehmi SM, Sultan S, Fishman DS, Qumseya BJ, Cortessis VK, Schilperoort H, Kysh L, Matsuoka L, Yachimski P, Agrawal D, Gurudu SR, Jamil LH, Jue TL, Khashab MA, Law JK, Lee JK, Naveed M, Sawhney MS, Thosani N, Yang J, Wani SB. ASGE guideline on the role of endoscopy in the evaluation and management of choledocholithiasis. Gastrointest Endosc. 2019;89(6):1075-1105.e15. http://doi.org/10.1016/j.gie.2018.10.001 